\title{
New role of bone morphogenetic protein 7 in brown adipogenesis and energy expenditure
}

\author{
Yu-Hua Tseng ${ }^{1,{ }^{*}}$, Efi Kokkotou ${ }^{3}$, Tim J. Schulz $^{1}$, Tian Lian Huang ${ }^{1}$, Jonathon N. Winnay ${ }^{1}$, \\ Cullen M. Taniguchi ${ }^{1}$, Thien T. Tran ${ }^{1}$, Ryo Suzuki ${ }^{1}$, Daniel O. Espinoza ${ }^{1}$, Yuji Yamamoto ${ }^{1}$, \\ Molly J. Ahrens ${ }^{5}$, Andrew T. Dudley ${ }^{5}$, Andrew W. Norris ${ }^{4}$, Rohit N. Kulkarni ${ }^{2}$, and C. Ronald \\ Kahn ${ }^{1}$ \\ ${ }^{1}$ Section on Obesity and Hormone Action, Joslin Diabetes Center, Harvard Medical School, Boston, \\ MA
}

${ }^{2}$ Section on Cell and Molecular Physiology, Joslin Diabetes Center, Harvard Medical School, Boston, MA

${ }^{3}$ Division of Gastroenterology, Beth Israel Deaconess Medical Center, Harvard Medical School, Boston, MA

${ }^{4}$ Division of Pediatric Endocrinology, University of lowa Children's Hospital, lowa City, IA

${ }^{5}$ Department of Biochemistry, Northwestern University, Evanston, IL

\begin{abstract}
Adipose tissue is central to the regulation of energy balance. Two functionally different types of fat are present in mammals: white adipose tissue (WAT), the primary site of triglyceride storage, and brown adipose tissue (BAT), which is specialized in energy expenditure and can counteract obesity ${ }^{1}$. Factors that specify the developmental fate and function of white and brown adipose tissue remain poorly understood ${ }^{2}, 3$. Here, we demonstrate that while some members of the family of bone morphogenetic proteins (BMP) support white adipocyte differentiation, BMP-7 singularly promotes differentiation of brown preadipocytes even in the absence of the normally required hormonal induction cocktail. BMP-7 activates a full program of brown adipogenesis including induction of early regulators of brown fat fate PRDM $16^{4}$ and PGC-1 (PPAR $\gamma$ coactivator-1) $\alpha^{5}$, increased expression of brown fat defining marker uncoupling protein-1 (UCP-1) and adipogenic transcription factors peroxisome proliferator-activated receptor (PPAR) $\gamma$ and CCAAT/enhancer-binding proteins (C/EBPs), and mitochondrial biogenesis via a p38 MAP kinase and PGC-1 dependent pathway. Moreover, BMP-7 triggers commitment of mesenchymal progenitor cells to a brown adipocyte lineage, and implantation of these cells into nude mice results in development of adipose tissue containing mostly brown adipocytes. BMP-7 knockout embryos show a marked paucity of brown fat and near complete absence of UCP-1 protein. Adenoviral-mediated expression of BMP-7 in mice results in a significant increase in brown, but not white, fat mass and leads to an increase in energy expenditure and reduced weight gain. These data reveal an important role of BMP-7 in promoting brown adipocyte differentiation and thermogenesis in vivo and in vitro, and provide a potential novel therapeutic approach for the treatment of obesity.
\end{abstract}

\footnotetext{
*To whom correspondence should be addressed: Yu-Hua Tseng, Ph.D., Joslin Diabetes Center, One Joslin Place, Boston, MA 02215, Phone: 617-735-1967, Fax: 617-732-2593, E-mail: yu-hua.tseng@joslin.harvard.edu.

Supplementary Information is linked to the online version of the paper

Author Information Reprints and permissions information is available at www.nature.com/reprints.
} 
BMPs are members of the transforming growth factor- $\beta$ superfamily and control multiple key steps of embryonic development and differentiation ${ }^{6}$. BMPs appear to have different roles in adipogenesis $^{7}$. Although certain BMPs, in particular BMP-2 and BMP-4, enhance white adipogenesis when assisted by a hormonal induction cocktail ${ }^{8,9}$, the role of BMPs on the differentiation and function of BAT or the balance between WAT and BAT is unknown. To this end, we studied the role of BMPs in the differentiation of brown preadipocytes ${ }^{10}$ and 3T3L1 white preadipocytes in the absence of other hormonal or chemical inducers. Treatment of these cells with BMP-2, -4, -6 and -7 markedly increased lipid accumulation of brown preadipocytes in culture even in the absence of normally required induction cocktail or thiazolidinediones (Fig. 1a). BMP-5 exhibited a weaker effect as compared to other BMPs, and BMP-3 had virtually no effect on brown fat differentiation. By contrast, under the same conditions, 3T3-L1 white preadipocytes exhibited little or no differentiation when treated with these BMPs.

In brown preadipocytes, while BMP-2, -4, -6, and -7 induced lipid accumulation to similar extents, BMP-7 was unique in that it markedly induced UCP-1 mRNA expression (Fig. 1b) to a level comparable to that achieved by standard induction protocols (Supplementary Table S1). In addition, expression of several other brown fat selective genes was significantly induced by BMP-7 (Supplementary Fig. S1). By contrast, BMP-4, an adipogenic factor for white fat ${ }^{9}$, suppressed expression of UCP-1 in these brown preadipocytes, despite its effect on lipid accumulation. Western blot analysis confirmed the specific effect of BMP-7 on induction of UCP-1 protein expression in brown, but not white, preadipocytes (Supplementary Fig. S2). Importantly, expression of PGC-1 $\alpha$ and UCP-1 was markedly induced by cAMP to 6- and 18fold, respectively, in BMP-7-treated cells (Fig. 1c), indicating the differentiated lipidcontaining cells induced by BMP-7 are bona fide brown adipocytes with a complete capacity to initiate the thermogenic program. BMPs are known to stimulate osteogenic differentiation by inducing osteogenic transcription factor Runx 2 expression ${ }^{11}$. In brown preadipocytes, BMP-2, -6, and -7 significantly inhibited Runx2 expression, while BMP-4 had no effect (Fig. 1b), suggesting that these BMPs function in brown fat precursors to promote adipogenesis and inhibit osteogenic differentiation. The specific effect of BMP-7 on brown preadipocyte differentiation was also verified in primary culture isolated from stromo-vascular fraction of interscapular BAT (Supplementary Fig S3).

Cellular responses to BMPs have been shown to be mediated by the formation of a heteooligomeric complex of the type 1 and type 2 BMP receptors (BMPRs). Two major signaling pathways, the Smad pathway and p38 MAP kinase (MAPK) pathway, confer most of the biological function of BMPs ${ }^{12}$. We found there is only subtle difference in expression levels of different BMPR isoforms between brown and white preadipocytes (Supplementary Fig. S4). Interestingly, while BMP-7 increased phosphorylation of Smad1/5/8 in both brown and white preadipocytes, robust activation of p38 MAPK and its downstream transcription factor ATF-2 following BMP-7 stimulation was observed only in brown preadipocytes, while being blunted or almost completely absent in the 3T3-L1 white preadipocytes (Fig. 1d).

To further investigate the role of p38 MAPK in BMP-7-induced brown adipogenesis. Three pharmacological inhibitors of p38 MAPK were added individually to medium 7 hours prior to and throughout BMP-7 treatment. After 10 days in culture, while none of these inhibitors had an effect on BMP-7-induced lipid accumulation (Supplementary Fig. S5), all of these drugs effectively blocked expression of UCP-1 protein induced by BMP-7 (Fig. 1e). p38 MAPK is known to regulate thermogenesis via nuclear coactivator PGC-1 ${ }^{13,14}$. Indeed, we found that BMP-7-induced UCP-1 expression was markedly diminished in brown preadipocytes deficient in both PGC-1 $\alpha$ and PGC-1 $\beta^{15}$ (Supplementary Fig. S6). Together, these data reveal an essential role for $\mathrm{p} 38$ MAPK and PGC-1 coactivators for BMP-7-induced thermogenic program in brown adipocytes, while they are dispensable for its effect on lipid accumulation. 
Before entering the adipogenic program, preadipocytes must be released from suppressive factors and become committed to terminal differentiation ${ }^{3}$. Necdin acts as a negative modulator of brown preadipocyte differentiation, coordinating early adipogenic events, including suppression of Pref-1 and Wnt10a expression ${ }^{16}$. Treatment of brown preadipocytes with BMP-7 significantly suppressed expression of necdin (Fig. 2a). In addition, BMP-7 also markedly suppressed expression of other inhibitors of adipogenesis, including Pref-1 and Wnt10a (Fig. 2a). As a consequence of release from suppression by BMP-7 treatment, these brown preadipocytes initiated the full transcriptional program of adipogenesis as shown by a significant increase in expression of PPAR $\gamma, \mathrm{C} / \mathrm{EBP} \alpha$ and aP2 (Fig. 2b). Importantly, BMP-7 robustly induced expression of PRDM16, a zinc-finger binding protein recently identified as an early regulator determining brown fat fate ${ }^{4}$, by 6.3 -fold at day 3 (Fig. 2c). This led to increased expression of other molecular characteristics of brown fat, including PGC-1 $\alpha$, PGC-1 $\beta$ (Fig. 2c) and UCP-1 (Fig. 1b).

Differentiation of brown fat is accompanied by mitochondrial biogenesis ${ }^{17}$. In the brown preadipocyte cell line, BMP-7 treatment significantly increased the expression of genes involved in mitochondrial biogenesis and function (Fig. 2c, 2d and Supplementary Fig. S1), including PGC- $1 \alpha$ and PGC-1 $\beta$ as well as nuclear respiratory factor (NRF)-1, mitochondrial transcription factor A (Tfam) and cytochrome $\mathrm{C}$. This coincided with a 5-fold increase in mitochondrial density in BMP-7-treated cells compared to control (Fig. 2e). Thus, BMP-7 activated a full program of brown adipogenesis by suppression of early adipogenic inhibitors, induction of factors determining brown fat fate, increased expression of adipogenic transcription factors and mitochondrial biogenesis.

BMPs are important in control of stem cell commitment to various lineages ${ }^{18}$. To determine if BMP-7 could also trigger commitment of the mesenchymal progenitor/stem cells into a brown adipocyte lineage, we treated the multipotent $\mathrm{C} 3 \mathrm{H} 10 \mathrm{~T} 1 / 2$ cells with BMP-7 for 3 days prior to treatment with a standard adipogenic differentiation cocktail ${ }^{10}$. Cells pretreated with BMP-7, but not vehicle, displayed a mature brown adipocyte phenotype with marked increases in lipid accumulation, and induction of the brown fat specific protein UCP-1 (Fig. 3a). Expression of specific markers indicated that the $\mathrm{C} 3 \mathrm{H} 10 \mathrm{~T} 1 / 2$ cells had become committed to the brown adipocyte lineage within 3 days of BMP-7 treatment (Fig. 3b). By this time point, BMP-7 pre-treatment had increased expression of $\mathrm{C} / \mathrm{EBP} \delta^{19}$, followed by increased expression of $\mathrm{C} / \mathrm{EBP} \beta, \mathrm{C} / \mathrm{EBP} \alpha, \mathrm{PPAR} \gamma$ and $\mathrm{aP} 2$ at later stage of differentiation, consistent with previously described gene patterns in committed white and brown preadipocytes ${ }^{20,21}$. Interestingly, BMP-7 pretreatment also caused a transient induction of PGC-1 $\alpha$ expression and a significant increase in expression of NRF-1 and Tfam, followed by a later increase of cytochrome C expression (Fig. 3c), indicating increased mitochondrial biogenesis in these BMP-7 pretreated cells during the course of brown adipogenesis.

To verify the cell culture findings in vivo, we implanted BMP-7-treated C3H10T1/2 cells subcutaneously into athymic nude mice in the sternal region. Six weeks after implantation, the BMP-7-treated cells developed into a fat pad containing a large number of multi-locular and UCP-1-positive brown adipocytes and a small portion of uni-locular white adipocytes, while no additional tissue was found in mice receiving cells treated with vehicle (Fig. 3d and Supplementary Fig. S7). Additionally, BMP-7, in concert with other differentiating agents, induced brown adipogenesis in two more primitive fibroblastic cells with no adipogenic character (Supplementary Fig. S8 and S9). Thus, BMP-7 triggers commitment of multipotent mesenchymal cells to the brown adipocyte lineage in both in vitro and ex vivo settings.

To determine the physiological necessity of BMP-7 for BAT development, we analyzed brown fat morphology and function in BMP-7 knockout mice. Since BMP-7 null mice die shortly after birth due to renal failure 22,23 , we focused our study in newborns and embryos. In rodents 
at these stages brown fat is already developed, while white fat is still not grossly visible. Strikingly, at birth BMP-7 knockout mice displayed a marked 50-70\% decrease in interscapular BAT mass compared with wild-type littermates, while the size of other internal organs, such as the liver, as well as the size of whole animal, were not altered (Fig. 4a). The decrease of BAT mass in BMP-7 knockout animals was also evident at embryonic stages. Cross-sections of 17.5 days postcoitum (dpc) embryos showed a dramatic decrease in brown fat mass, due to a marked decrease in the number of brown adipocytes (Fig. $4 \mathrm{~b}$ and Supplementary Fig. S10). Most importantly, expression of UCP-1 protein was markedly decreased or completely absent in brown fat from 18.5 dpc BMP-7 null embryos, while expression of insulin receptor, another protein involved in adipogenesis, remained unaltered (Fig. 4c). This was accompanied with by a significant decrease in expression of a number of brown fat selective genes (Supplementary Figure S11). These data establish an essential role of BMP-7 in brown fat development in vivo and a near absolute requirement for BMP-7 in maintaining brown fat specific thermogenic program.

Finally to explore the potential role of BMP-7 in regulation of brown adipogenesis and energy homeostasis in vivo, we injected adenoviruses expressing BMP and LacZ control via the tail vein into 4-week old C57BL/6 mice and measured metabolic rate by indirect calorimetry. Adenoviruses are trophic for the liver, where they can drive release of secretory proteins, like BMPs, into the blood stream (Supplementary Fig. S12a). Importantly, mice that received adenovirus expressing BMP-7 showed significant increases in whole-body energy expenditure and basal body temperature, leading to a significant reduction in weight gain as compared with mice that received LacZ adenovirus (Fig. 4d). The increase in energy expenditure in BMP-7treated mice was not due to an increase in physical activity or food intake (Supplementary Fig. S12). By contrast, BMP-3, which had no effect on differentiation of brown preadipocytes in vitro (Fig. 1a), did not produce any differences compared to control mice. Fifteen days after adenoviral injection, mice that received BMP-7 treatment displayed a significant increase in brown fat mass with no change in WAT mass (Supplementary Fig. S13). In a cohort of older mice, BMP-7 treatment specifically induced expression of PRDM16 and UCP-1, two key factors determining brown fat fate and function, in BAT (Fig. 4e), while the expression of genes involved in energy homeostasis in other tissues, including WAT, muscle and liver, remained unaltered (Supplementary Fig. S14). Together, these data not only recapitulate the brown adipogenic effect of BMP-7 in vivo, but also reveal an important anti-obesity potential of BMP-7 via increasing whole body thermogenesis.

BAT and WAT are morphologically and functionally different tissues, and their developmental patterns are quite distinct. One of the remaining questions in adipocyte biology is how and when the developmental fate of brown versus white adipocytes is regulated and specified. On the basis of the present data and published observations from other investigators $8,9,24$, we propose a model for the role of BMPs in determination of brown versus white fat cell fate, as illustrated in Fig. 4f. While BMP-2 and BMP-4 can promote differentiation of white adipocyte lineage, we demonstrate in the present study that BMP-7 drives brown fat cell fate in both mesenchymal progenitor cells and committed brown preadipocytes. This is achieved by suppression of early adipogenic inhibitors, such as necdin, Pref-1 and Wnts, and by induction of key molecules that specify brown fat fate, such as PRDM16 and PGC-1 $\alpha$, leading to a mature brown adipocyte phenotype with UCP-1 expression and abundant mitochondria. BMP-7 null embryos display brown fat hypoplasia and almost complete absence of UCP-1 protein, highlighting an essential role of BMP-7 in brown fat development. When applied in vivo, BMP-7 is able to increase brown fat mass and thermogenic energy expenditure in mice.

Whereas BMP-2, -4, -6, and -7 are able to induce massive lipid accumulation in brown preadipocytes, we find that only BMP-7 has a specific effect on induction of the brown fat specific protein UCP-1. Differential effects of various BMPs on other single cell types have 
also been observed ${ }^{25}$. For example, BMP-7, but not BMP-4 or BMP-6, is able to reverse TGF$\beta$-induced epithelial-to-mesenchymal transition in distal tubular epithelial cells ${ }^{26}$. Exactly how the specificity of different BMPs is determined remains an unsolved question in the field. Our data have indicated an important role of p38 MAPK and PGC-1 coactivator in BMP-7-induced thermogenesis. Originally identified as a bone inducer ${ }^{27}$, BMP-7 is now being recognized as a multifunctional cytokine and has been implicated as a potential therapeutic agent for cardiovascular, metabolic and degenerative diseases ${ }^{28}$. In this study, our data reveal a novel function of BMP-7 in the regulation of energy homeostasis by promoting brown, but not white, fat differentiation and function. Thus, we propose that treatment of humans with BMP-7 or its molecular mimetic may recruit and activate brown fat differentiation, leading to an increase in energy expenditure, and thereby providing a new avenue to combat obesity.

\section{Methods Summary}

\section{Adipocyte Differentiation}

To induce adipocyte differentiation by BMPs in the absence of induction cocktails, both WT brown preadipocytes and 3T3-L1 white preadipocytes were grown in regular growth medium supplemented with combination of rhBMPs (3.3 to $8.3 \mathrm{nM})$, insulin $(20 \mathrm{nM})$ and T3 (1 nM) or vehicle as indicated in the text and figure legends for 7-13 days. To stimulate thermogenic program, differentiated cells were incubated with $500 \mu \mathrm{M}$ dibutyrul cyclic AMP for 4 hrs. Cells were grown in growth medium without hormonal supplements for $18 \mathrm{hrs}$ prior to cAMP stimulation.

C3H10T1/2 cells were grown in the presence and absence of $8.3 \mathrm{nM}$ rhBMP-7 for 3 days to reach confluence (day 3 ). These cells were then induced to adipocyte differentiation using protocols described below for additional 7 days (day 10). Adipocyte differentiation was done by treating confluent cells for 48 hours in medium supplemented with $20 \mathrm{nM}$ insulin and $1 \mathrm{nM}$ triiodothyronine (T3), $0.5 \mathrm{mM}$ isobutylmethylxanthine (IBMX), $5 \mu \mathrm{M}$ dexamethasone, and $0.125 \mathrm{mM}$ indomethacin. Cells were placed back to growth medium supplemented with insulin and T3, which was then changed every second day. After four to five more days in this medium, cells exhibited a fully differentiated phenotype with massive lipid accumulation.

\section{Full Methods}

\section{Materials}

Recombinant human BMPs (rhBMPs) were purchased from R\&D Systems (Minneapolis, $\mathrm{MN})$. Antibodies used for immunoblotting included anti-UCP-1, anti-PPAR $\gamma$, anti- $\beta$-tubulin (all purchased from Santa Cruz Biotechnology, Santa Cruz, CA), anti-FAS (a generous gift from Dr. F. P. Kuhajda, Johns Hopkins Medical Institute, Baltimore, MD), anti-phosphoSmad1/5/8, anti-phospho-p38 MAPK, anti-phospho-ATF-2, anti- $\beta$-tubulin, anti-insulin receptor $\beta$ subunit (Cell Signaling) and anti-cyclophilin A (Upstate Biotechnology, Lake Placid, NY). Immobolin-P transfer membranes were from Millipore (Billerica, MA) and electrophoresis supplies were from Bio-Rad Laboratories (Hercules, CA.). SB202190, SB203280, and PD169316 were purchased from Calbiochem (San Diego, CA) and dissolved in DMSO. All other chemicals were purchased from Sigma Chemical Co. (St. Louis, MO.) unless otherwise specified.

\section{Cell Culture}

C3H10T1/2 and 3T3-L1 cells were purchased from American Type Culture Collection (Manassas, VA). Generation of WT brown preadipocyte cell lines that derived from newborn wild-type mice was as described previously $10,29-31$. PGC-1 null brown preadipocyte cell line was a generous gift from Dr. Spiegelman (Dana Farber Cancer Institute, Boston, MA) ${ }^{15}$. All 
cell lines used in this study were maintained in Dulbecco's modified Earle's medium (DMEM) $10 \%$ Fetal Clone III (HyClone, Logan, VT) at $37^{\circ} \mathrm{C}$ in $5 \% \mathrm{CO}_{2}$ environment unless otherwise specified.

\section{Quantitative-Reverse Transcription-Polymerase Chain Reaction (Q-RT-PCR) analysis}

Total RNA was isolated with QIAzol lysis reagent (Qiagen, Valencia, CA) and purified by RNeasy Mini columns (Qiagen) following the manufacture's instructions. cDNA was prepared from $1 \mu \mathrm{g}$ of RNA using the Advantage RT-PCR kit (BD Biosciences, Palo Alto, CA) according to manufacturer's instructions and diluted to a final volume of $250 \mu 1.5 \mu 1$ of diluted cDNA was used in a $20 \mu \mathrm{l} \mathrm{PCR}$ reaction with SYBR Green Master Mix (Applied Biosystems, Foster City, CA) and primers at a concentration of $300 \mathrm{nM}$ each. PCR reactions were run in duplicate for each sample and quantitated in the ABI Prism 7000 Sequence Detection System (Applied Biosystems). Data were expressed as arbitrary units after normalization to levels of expression of internal controls Acidic Ribosomal Phosphoprotein P0 (Arbp, 36B4) for each sample. Sequences of primers used in this study are listed in Supplementary Table 1.

\section{Oil red $O$ staining}

Dishes were washed twice with phosphate-buffered saline and fixed with $10 \%$ buffered formalin overnight at $4^{\circ} \mathrm{C}$. Cells were then stained for 2 hours at room temperature with a filtered oil red $\mathrm{O}$ solution ( $0.5 \%$ oil red $\mathrm{O}$ in isopropyl alcohol), washed twice with distilled water, and visualized.

\section{Western blot analysis}

Cells were harvested in lysis buffer (50 mM HEPES, $137 \mathrm{mM} \mathrm{NaCl}, 1 \mathrm{mM} \mathrm{MgCl} 2,1 \mathrm{mM}$ $\mathrm{CaCl}_{2}, 10 \mathrm{mM} \mathrm{Na}_{2} \mathrm{P}_{2} \mathrm{O}_{7}, 10 \mathrm{mM} \mathrm{NaF}, 2 \mathrm{mM}$ EDTA, $10 \%$ glycerol, $1 \%$ Igepal CA-630, $2 \mathrm{mM}$ vanadate, $10 \mu \mathrm{g} / \mathrm{ml}$ of leupeptin, $10 \mu \mathrm{g} / \mathrm{ml}$ of aprotinin, $2 \mathrm{mM}$ phenylmethylsulfonyl fluoride; $\mathrm{pH}$ 7.4). After lysis, lysates were clarified by centrifugation at $12,000 \times \mathrm{g}$ for $20 \mathrm{~min}$ at $4^{\circ} \mathrm{C}$, the protein amount in the supernatants was determined by the Bradford Protein Assay (BioRad Laborotories, Hercules, CA). Proteins were directly solubilized in Laemmli sample buffer. Equal amounts of lysates were separated by sodium dodecyl sulfate-polyacrylamide gel electrophoresis and transferred to Immobolin-P membranes. Membranes were blocked overnight at $4{ }^{\circ} \mathrm{C}$ and incubated with the indicated antibody for 2 hours at room temperature. Specifically bound primary antibodies were detected with peroxidase-coupled secondary antibody and enhanced chemiluminescence (ECL, Amersham Biosciences, Piscataway, NJ).

\section{Electron Microscopy}

Cells were fixed in $2.5 \%$ glutaraldehyde, then post-fixed in $2 \%$ osmium teroxide, dehydrated in ascending gradations of ethanol, and embedded in fresh Araldite 502 epoxy resin using BEEM capsules. Ultra-thin sections $(60-80 \mathrm{~nm})$ were cut and mounted on 75 mesh copper grids, then stained with uranyl acetate and lead citrate before being examined on the Phillips 301 transmission eletron microscope. Mitochondrial and total cytoplasmic areas were quantified by using the the NIH Image J software (http://rsb.info.nih.gov/ij/). Mitochondrial density were determined by the ratio of the sum of mitochondrial area to total cytoplasmic area per cell.

\section{Isolation of Stromo-Vascular Fractions (SVF) and In vito Differentiation}

Eight 6-week old C57BL/6 male mice were sacrificed. Interscapular BAT and axillary subcutaneous WAT were removed, minced and digested with $1 \mathrm{mg} / \mathrm{ml}$ collagenase for $45 \mathrm{~min}$ at $37^{\circ} \mathrm{C}$ in DMEM/F12 medium, containing $1 \%$ BSA and antibiotics. Digested tissues were filtered through sterile $150 \mu \mathrm{m}$ nylon mesh and centrifuged at $250 \times \mathrm{g}$ for $5 \mathrm{~min}$. The floating fractions consisting of adipocytes were discarded and the pellets representing the SVF were 
then resuspended in erythrocyte lysis buffer $\left(154 \mathrm{mM} \mathrm{NH}_{4} \mathrm{Cl}, 10 \mathrm{mM} \mathrm{KHCO}_{3}, 0.1 \mathrm{mM}\right.$ EDTA) for $10 \mathrm{~min}$ to remove red blood cells. The cells were further centrifuged at $500 \times \mathrm{g}$ for $5 \mathrm{~min}$, plated at $8 \times 10^{5} /$ well of a 24 -well plate and grown at $37^{\circ} \mathrm{C}$ in DMEM/F12 supplemented with $10 \% \mathrm{FBS}$ at $37^{\circ} \mathrm{C}$.

In vitro differentiation was performed using the method described by Hauner et al. ${ }^{32}$. After 2 days of incubation, the attached cells were washed and incubated in serum free differentiation medium, containing DMEM/F12 medium supplemented with $1 \mu \mathrm{M}$ dexamethasone, $66 \mathrm{nM}$ insulin, $15 \mathrm{mmol} / \mathrm{l} \mathrm{HEPES}, 1 \mathrm{nM} \mathrm{T} 3,33 \mu \mathrm{M}$ biotin, $17 \mu \mathrm{M}$ pantothenate, $10 \mu \mathrm{g} / \mathrm{ml}$ transferrin, and $100 \mu \mathrm{g} / \mathrm{ml}$ penicillin-streptomycin in the absence or presence of $1 \mu \mathrm{g} / \mathrm{ml}$ rosiglitazone or $3.3 \mathrm{nM}$ rhBMP-7 for 3 days.

\section{Implantation}

C3H10T1/2 cells were grown in the presence and absence of $3.3 \mathrm{nM}$ rhBMP-7 for 3 days to reach confluence. Cells were washed, trypsinzed, and resuspended in growth medium. $1.5 \times$ $10^{7}$ cells in $0.15 \mathrm{ml}$ volume were injected into the thoracic/sternum region of 5-w old BALB/ $\mathrm{c}$ athymic mice (Charles River Laboratories, Inc., Wilmington, MA) using a 18-gauge needle. Mice were sacrificed at 6 weeks after injection and adipose tissue derived from implanted cells were excised and proceeded for histological analysis.

\section{Histology and Immunohistochemistry}

Tissues were fixed in 10\% formalin and paraffin-embedded. Multiple sections were prepared and stained with H\&E for general morphological observation. UCP-1 immunohistochemistry of tissue from implanted cells was performed using polyclonal anti-mouse UCP-1 antibody

(Chemicon International Inc., Temecula, CA) at 1:50 dilution and the Dako Envision Doublestain System (Dako, Carpinteria, CA) following the manufacture's instruction. Slides were counterstained with Hematoxylin.

\section{Genotyping of BMP-7 Null Mice}

BMP-7 null mice were generated by intercrosses of animals heterozygous for a null allele of BMP-7 generated by insertion of lacZ into exon $1^{33}$. Genotypes were initially determined by a combination of $\beta$-galactosidase staining and the presence or absence of a severe eye phenotype (very small eyes or no eye) $22,23,33$. All genotypes were confirmed by PCR as described by Bandyopadhyay et al. ${ }^{34}$. For $\beta$-galactosidase staining, the tips of tails were clipped, fixed in $4 \%$ PFA plus $2 \mathrm{mM} \mathrm{MgCl} 2$ in PBS for $30 \mathrm{~min}$ at room temperature. The tails were washed in $2 \mathrm{mM} \mathrm{MgCl} 2$ in PBS $3 \times 10 \mathrm{~min}$ at room temperature. The tails were stained at $37^{\circ} \mathrm{C}$ overnight in $\mathrm{X}$-gal staining solution $(5 \mathrm{mM}$ potassium ferrocyanide, $5 \mathrm{mM}$ potassium ferricyanide, $2 \mathrm{mM} \mathrm{MgCl} 2,0.5 \mathrm{mg} / \mathrm{ml}$ 5-bromo-4-chloro-3-indolyl-b-D-galactosidase X-gal, Denville Scientific, Inc.). The presence of $\beta$-galactosidase staining in the tails indicates that the lacZ transgene is present in BMP-7 exon 1. A lack of $\beta$-galactosidase staining indicates the mouse is wild-type.

\section{Adenoviral Injection}

Adenoviruses were amplified in HEK293 cells as previously described ${ }^{35}$. Prior to in vivo use, all adenoviruses were purified on a cesium chloride gradient and dialyzed into PBS plus $10 \%$ glycerol. Four and twelve-week-old male C57BL/6 mice were injected via tail vein with a adenoviral dose $5 \times 10^{8}$ viral particle/g body weight as described previously ${ }^{36}$. Mice were sacrificed at 15 days after injection. Intrerscapular brown fat and epididymal white fat were excised and weighted. Half of the tissue was fixed in $10 \%$ formalin and proceed for histological analysis. The other half of the tissue was subjected for RNA extraction and Q-RT-PCR analysis. 


\section{Indirect Calorimetry}

Metabolic rates were measured by indirect calorimetry in mice 7-10 days after adenoviral injection by using the Comprehensive Lab Animal Monitoring System (CLAMS, Columbus Instruments, Columbus, OH). Mice were maintained at $24^{\circ} \mathrm{C}$ under a 12 -h light/dark cycle. Food and water are available ad libitum. Mice were acclimatized to individual cages for $24 \mathrm{~h}$ before recording, and then underwent $24 \mathrm{~h}$ of monitoring.

Heat production (energy expenditure) was calculated using the following equations:

Heat $[\mathrm{kcal} / \mathrm{hr}]=\left(3.815+1.232 *\left(\mathrm{VCO}_{2} / \mathrm{VO}_{2}\right)\right) * \mathrm{VO}_{2}[$ liter $/ \mathrm{kg} / \mathrm{hr}] *$ body weight $[\mathrm{kg}]$

\section{Measurement of plasma BMP-7 concentrations}

Blood was collected at day 1 and day 3 after adenoviral injection and at the time of sacrifice. Plasma BMP-7 levels were determined by ELISA assay using the DuoSet ELISA Development kit purchased from R\&D Systems following the manufacture's instructions. Concentrations were calculated using a standard curve generated by rhBMP-7 standards included in the kit.

\section{Statistical Analysis}

Statistical significance in gene expression between control and BMP-treated group was determined by analysis of variance (ANOVA) test or Student's $t$ test unless otherwise specified. To evaluate if there was a significant change in body weight between BMP-7 and control groups, we performed multivariate analysis of variance (MANOVA) test. Based on multivariate normal assumption on the data, Hotelling's $T^{2}$ statistics was calculated and yielded $T^{2}=3.9613$ and a significant $p$-value of 0.0191 . The $T^{2}$ test of Harold Hotelling compares means of two or more continuous measures simultaneously for the two groups. Considering the small size of samples and the validity of multivariate normal assumption, we also performed the sign and rank based MANOVA analyses. The sign based MANOVA gave $p$-value of 0.0581 and the rank based MANOVA yielded a $p$-value of 0.0612 . Both are marginally statistically significant. Comparison was also conducted at different time point individually. We found that at day 5 after adenoviral injection, the percentage of body weight changes relative to the initial body weight was significant between the control and BMP-7 groups ( $p$-value $=0.0469$ by Wilcoxon Signed Rank test).

\section{Supplementary Material}

Refer to Web version on PubMed Central for supplementary material.

\section{References}

1. Gesta S, Tseng YH, Kahn CR. Developmental origin of fat: tracking obesity to its source. Cell 2007;131:242-256. [PubMed: 17956727]

2. Farmer SR. Transcriptional control of adipocyte formation. Cell Metab 2006;4:263-273. [PubMed: 17011499]

3. Rosen ED, MacDougald OA. Adipocyte differentiation from the inside out. Nat. Rev. Mol Cell Biol 2006;7:885-896. [PubMed: 17139329]

4. Seale P, et al. Transcriptional Control of Brown Fat Determination by PRDM16. Cell Metab 2007;6:3854. [PubMed: 17618855]

5. Puigserver $P$, et al. A cold-inducible coactivator of nuclear receptors linked to adaptive thermogenesis. Cell 1998;92:829-839. [PubMed: 9529258]

6. Chen D, Zhao M, Mundy GR. Bone morphogenetic proteins. Growth Factors 2004;22:233-241. [PubMed: 15621726]

7. Tseng YH, He TC. Bone Morphogenetic Proteins and Adipocyte Differentiation. Cellscience Review 2007;3:342-360. 
8. Wang EA, Israel DI, Kelly S, Luxenberg DP. Bone morphogenetic protein-2 causes commitment and differentiation in C3H10T1/2 and 3T3 cells. Growth Factors 1993;9:57-71. [PubMed: 8347351]

9. Tang QQ, Otto TC, Lane MD. Commitment of C3H10T1/2 pluripotent stem cells to the adipocyte lineage. Proc Natl. Acad Sci U S. A 2004;101:9607-9611. [PubMed: 15210946]

10. Klein J, et al. Beta(3)-adrenergic stimulation differentially inhibits insulin signaling and decreases insulin induced glucose uptake in brown adipocytes. J. Biol. Chem 1999;274:34795-34802. [PubMed: 10574950]

11. Ducy P, Zhang R, Geoffroy V, Ridall AL, Karsenty G. Osf2/Cbfa1: a transcriptional activator of osteoblast differentiation. Cell 1997;89:747-754. [PubMed: 9182762]

12. Canalis E, Economides AN, Gazzerro E. Bone morphogenetic proteins, their antagonists, and the skeleton. Endocr. Rev 2003;24:218-235. [PubMed: 12700180]

13. Puigserver $P$, et al. Cytokine stimulation of energy expenditure through p38 MAP kinase activation of PPARgamma coactivator-1. Mol Cell 2001;8:971-982. [PubMed: 11741533]

14. Cao W, et al. p38 mitogen-activated protein kinase is the central regulator of cyclic AMP-dependent transcription of the brown fat uncoupling protein 1 gene. Mol Cell Biol 2004;24:3057-3067. [PubMed: 15024092]

15. Uldry M, et al. Complementary action of the PGC-1 coactivators in mitochondrial biogenesis and brown fat differentiation. Cell Metab 2006;3:333-341. [PubMed: 16679291]

16. Tseng YH, et al. Prediction of preadipocyte differentiation by gene expression reveals role of insulin receptor substrates and necdin. Nat. Cell Biol 2005;7:601-611. [PubMed: 15895078]

17. Nedergaard, J.; Connally, E.; Cannon, B. Brown Adipose Tissue. Trayhurn, P.; Nicholls, DG., editors. Baltimore: Edward Arnold; 1986. p. 152-213.

18. Varga AC, Wrana JL. The disparate role of BMP in stem cell biology. Oncogene 2005;24:5713-5721. [PubMed: 16123804]

19. Darlington GJ, Ross SE, MacDougald OA. The role of C/EBP genes in adipocyte differentiation. J Biol Chem 1998;273:30057-30060. [PubMed: 9804754]

20. Wu Z, et al. Cross-regulation of C/EBP alpha and PPAR gamma controls the transcriptional pathway of adipogenesis and insulin sensitivity. Mol Cell 1999;3:151-158. [PubMed: 10078198]

21. Tseng YH, Kriauciunas KM, Kokkotou E, Kahn CR. Differential roles of insulin receptor substrates in brown adipocyte differentiation. Mol Cell Biol 2004;24:1918-1929. [PubMed: 14966273]

22. Dudley AT, Lyons KM, Robertson EJ. A requirement for bone morphogenetic protein-7 during development of the mammalian kidney and eye. Genes. Dev 1995;9:2795-2807. [PubMed: 7590254]

23. Luo G, et al. BMP-7 is an inducer of nephrogenesis, and is also required for eye development and skeletal patterning. Genes. Dev 1995;9:2808-2820. [PubMed: 7590255]

24. Jin W, et al. Schnurri-2 controls BMP-dependent adipogenesis via interaction with Smad proteins. Dev Cell 2006;10:461-471. [PubMed: 16580992]

25. Cheng H, et al. Osteogenic activity of the fourteen types of human bone morphogenetic proteins (BMPs). J Bone Joint Surg. Am 2003;85-A:1544-1552. [PubMed: 12925636]

26. Zeisberg M, et al. BMP-7 counteracts TGF-beta1-induced epithelial-to-mesenchymal transition and reverses chronic renal injury. Nat. Med 2003;9:964-968. [PubMed: 12808448]

27. Ozkaynak E, et al. OP-1 cDNA encodes an osteogenic protein in the TGF-beta family. EMBO J 1990;9:2085-2093. [PubMed: 2357959]

28. Tobin JF, Celeste AJ. Bone morphogenetic proteins and growth differentiation factors as drug targets in cardiovascular and metabolic disease. Drug Discov. Today 2006;11:405-411. [PubMed: 16635802]

29. Fasshauer M, et al. Essential role of insulin receptor substrate-2 in insulin stimulation of glut4 translocation and glucose uptake in brown adipocytes. J Biol Chem 2000;275:25494-25501. [PubMed: 10829031]

30. Fasshauer M, et al. Essential role of insulin receptor substrate 1 in differentiation of brown adipocytes. Mol Cell Biol 2001;21:319-329. [PubMed: 11113206]

31. Tseng YH, Ueki K, Kriauciunas KM, Kahn CR. Differential roles of insulin receptor substrates in the anti-apoptotic function of insulin-like growth factor-1 and insulin. J Biol. Chem 2002;277:3160131611. [PubMed: 12082100] 
32. Hauner H, et al. Promoting effect of glucocorticoids on the differentiation of human adipocyte precursor cells cultured in a chemically defined medium. J Clin Invest 1989;84:1663-1670. [PubMed: 2681273]

33. Godin RE, Takaesu NT, Robertson EJ, Dudley AT. Regulation of BMP7 expression during kidney development. Development 1998;125:3473-3482. [PubMed: 9693150]

34. Bandyopadhyay A, et al. Genetic analysis of the roles of BMP2, BMP4, and BMP7 in limb patterning and skeletogenesis. PLoS. Genet 2006;2:e216. [PubMed: 17194222]

35. Ueki K, Kondo T, Tseng YH, Kahn CR. Central role of suppressors of cytokine signaling proteins in hepatic steatosis, insulin resistance, and the metabolic syndrome in the mouse. Proc Natl. Acad Sci U S. A 2004;101:10422-10427. [PubMed: 15240880]

36. Laustsen PG, et al. Lipoatrophic diabetes in Irs1(-/-)/Irs3(-/-) double knockout mice. Genes. Dev 2002;16:3213-3222. [PubMed: 12502742]

\section{Acknowledgments}

We acknowledge T.-C. He (University of Chicago Medical Center) for providing adenoviruses expressing BMPs. We thank M. Rourk and L. Mazzola for help with the animal experiments and J. Hu, C. Cahill, A. McSweeney, L. Polivy (Joslin's DERC Core), and R. Bronson (Rodent Histopathology Core, Harvard Medical School) for technical assistance, and P. Zhang (Harvard School of Public Health) for statistical consultation. We thank A. Butte and P. Laustsen for inputs on initiation of this project. We thank M. Uldry and B. Spiegelman (Dana-Farber Cancer Institute) for providing the PGC-1 null cells. This work was supported in part by the National Institutes of Health grants R01 DK077097, R21 DK070722, P30 DK46200, and P30 DK040561 (to Y.-H. T.), R01 DK67536 (to R. N. K.), K08 DK64906 (to A.W.N.) and R01 DK 060837 (to C. R. K), the Tanita Healthy Weight Community the Eleanor and Miles Shore $50^{\text {th }}$ Anniversary Scholar Program from Harvard Medical School (to Y.-H. T.). 
a Control BMP-2 BMP-3 BMP-4 BMP-5 BMP-6 BMP-7

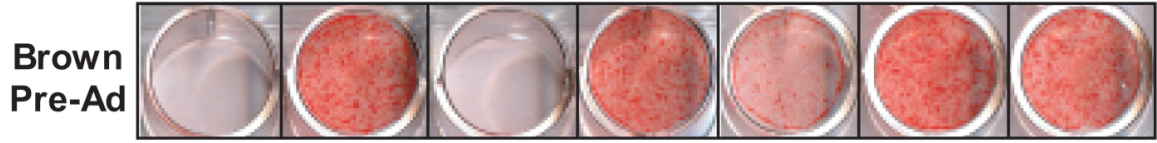

White

Pre-Ad

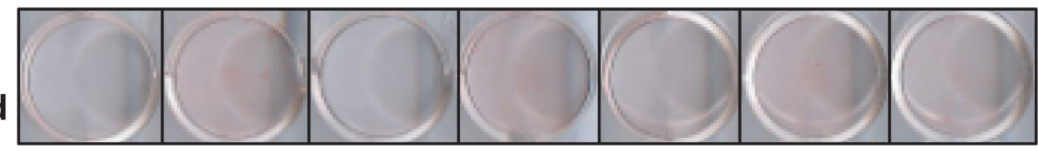

b

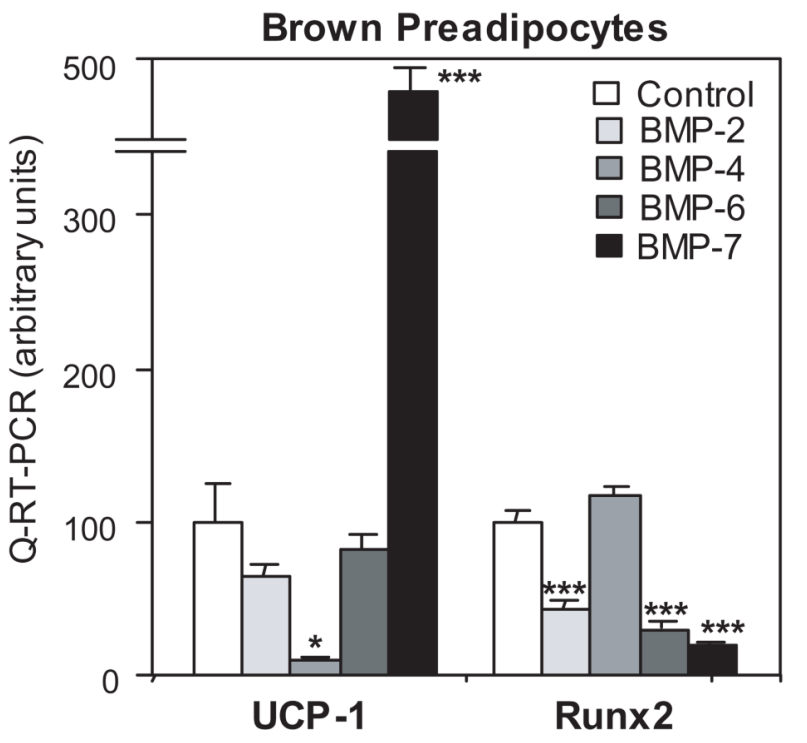

C BMP-7-Tx Brown Pre-Ad

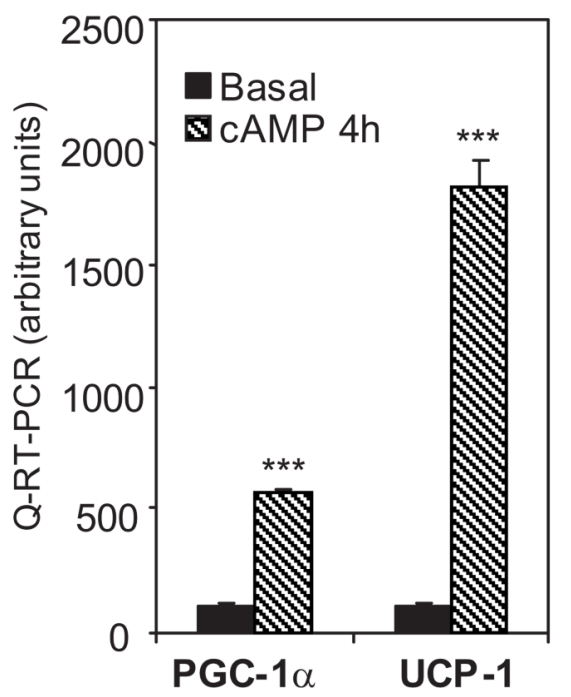

e

DMSO SB202190 SB203280 PD169316

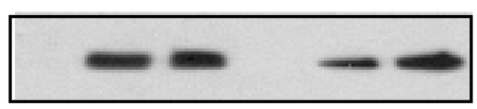

Phos.

Smadi $1 / 5 / 8$
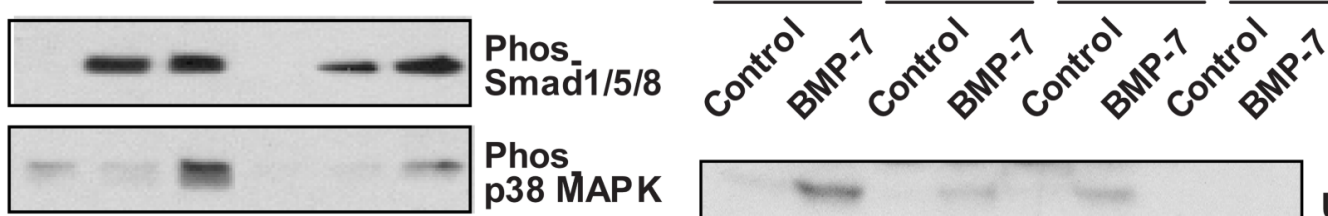

Phos
p38 MAPK

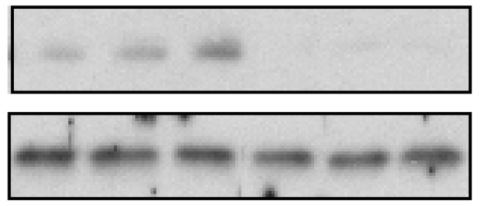

Phos-

ATF-2

Cур A

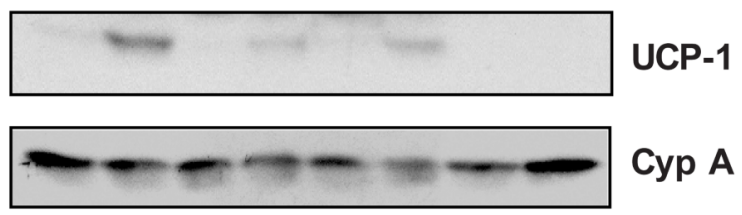

Figure 1. BMP-7 induces brown, but not white, preadipocyte differentiation and the essential role of 38 MAPK in BMP-7-induced thermogenesis

a, Oil Red O staining of brown preadipocytes and 3T3-L1 white preadipocytes grown in growth medium supplemented with BMPs or vehicle (control) for 8 days. b, Quantitative-RT-PCR (Q-RT-PCR) analysis for UCP-1 and Runx2 in brown preadipocytes treated with vehicle or BMPs in combination of insulin and T3 for 7 days. c, Q-RT-PCR analysis for PGC-1 $\alpha$ and UCP-1 in response to $4 \mathrm{hrs}$ of cAMP stimulation in brown preadipocytes differentiated in growth medium supplemented with BMP-7. Data are presented as mean $\pm \operatorname{SEM}(n=3)$. Asterisks depict statistically significant differences between control and experimental groups (*P<0.05, ** $P<0.01$, *** $P<0.001)$. d, Western blot analysis of phosphorylation of 
Smad1/5/8, p38 MAPK, and ATF-2 in response to 0, 10, and 30 min of BMP-7 stimulation in brown and white preadipocytes. e, Western blot analysis of UCP-1 in brown preadipocytes cultured in growth medium supplemented with vehicle or BMP-7 for 10 days. Three p38 MAPK inhibitors or vehicle (DMSO) were added to the cells $7 \mathrm{hrs}$ prior to and throughout BMP-7 treatment. Cyclophilin A (Cyp A) serves as a loading control. 
a

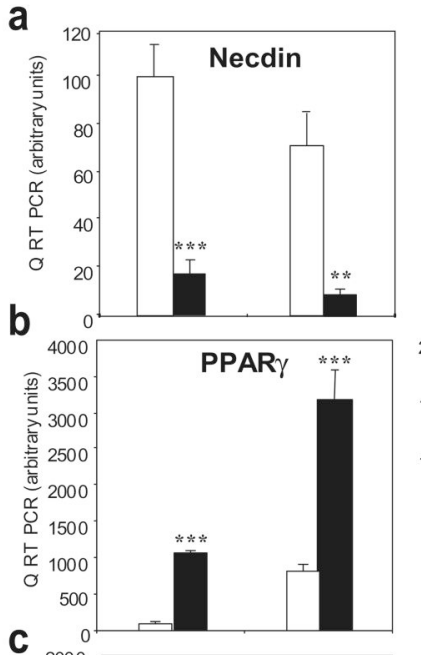

C

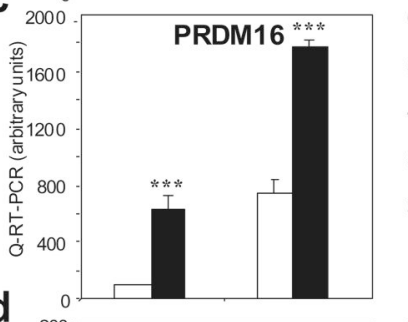

d

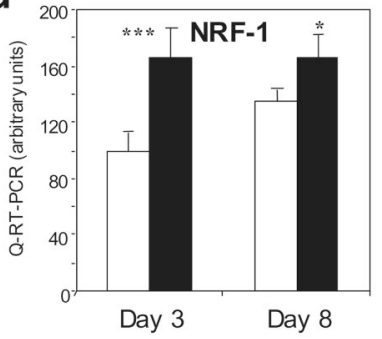

e

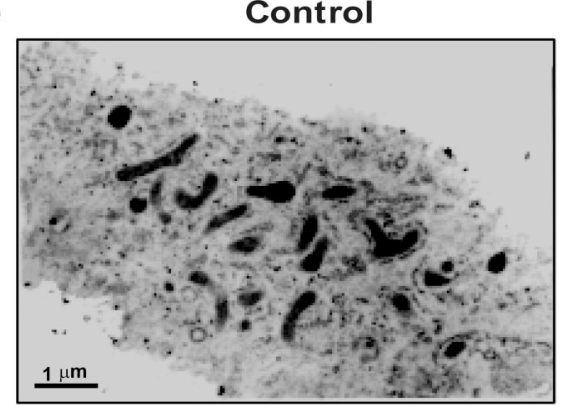

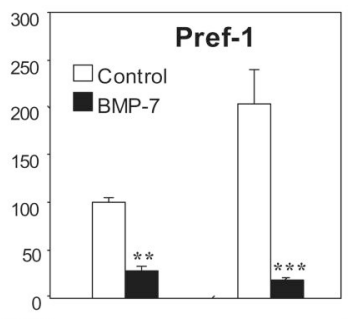
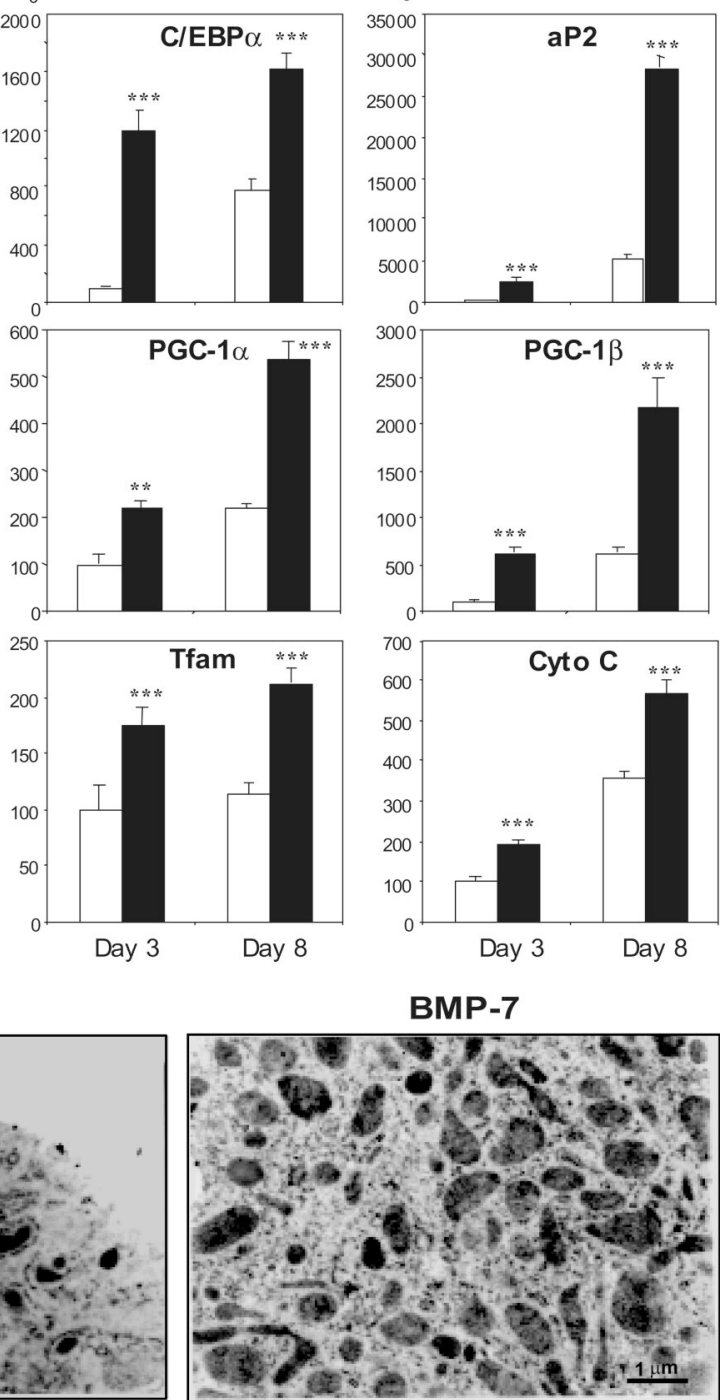

Figure 2. Molecular mechanisms by which BMP-7 induces brown adipogenesis and mitochondrial biogenesis

Q-RT-PCR analysis for genes served as (a) early adipogenic inhibitors, (b) adipogenic markers common to brown and white fat, (c) brown fat-specific markers, and (d) mitochondrial components in brown preadipocytes treated with vehicle (control) or BMP-7 for 3 or 8 days. Data are presented as mean \pm SEM $(\mathrm{n}=3)$. ( ${ }^{*} P<0.05$, ** $P<0.01$, *** $\left.P<0.001\right)$. e,

Transmission electron microscopy of brown preadipocytes treated with vehicle or BMP-7 for 9 days. Original magnification $=24,000 \mathrm{X}$. 
a

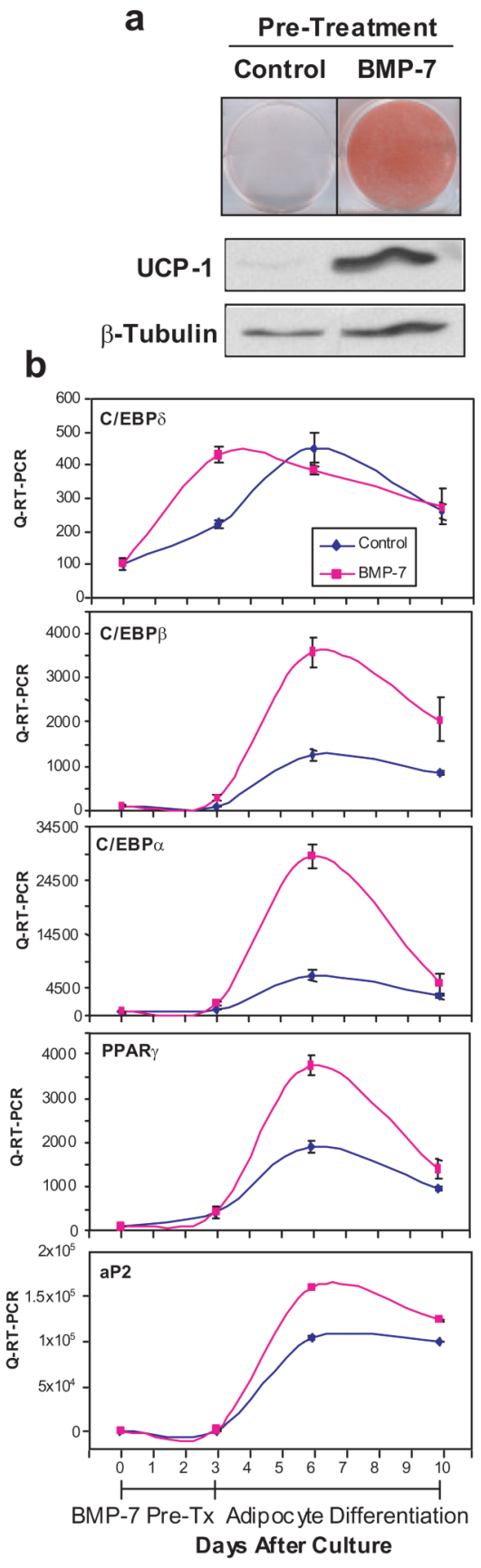

d

BMP-7-treated Implant
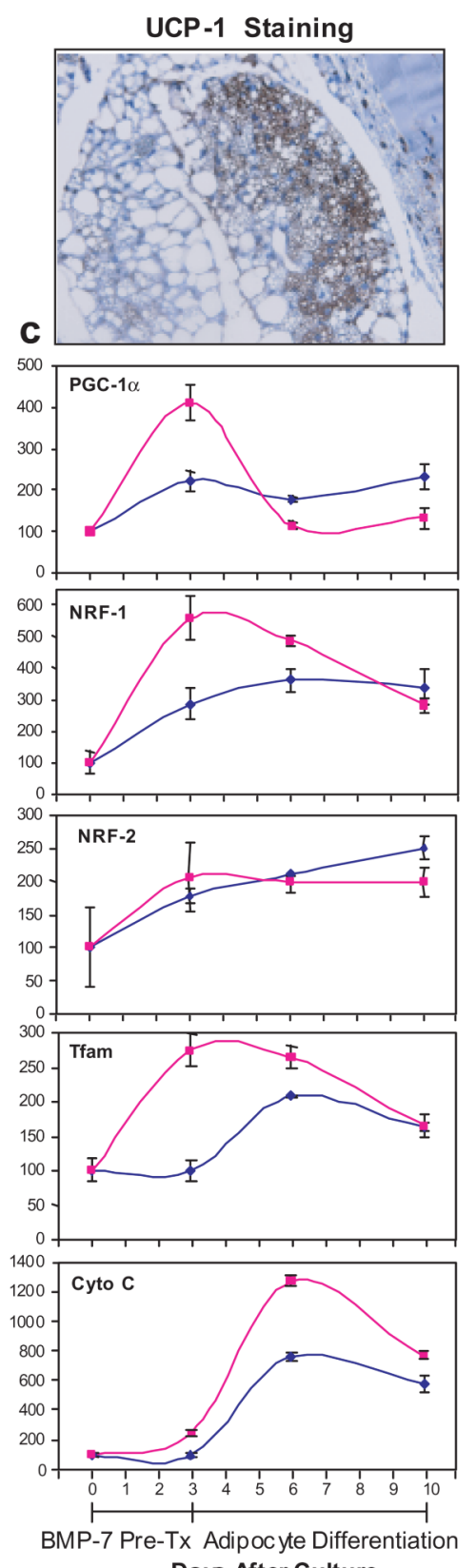

Days After Culture

Figure 3. BMP-7 triggers commitment of mesenchymal progenitor cells to brown adipocyte lineage in vitro and in vivo

a, Oil Red O staining and Western blotting analysis for UCP-1 in C3H10T1/2 cells treated with BMP-7 or vehicle (control) for 3 days followed by adipogenic induction for 7 days. $\beta$ tubulin serves as a loading control. b, c, Q-RT-PCR analysis for genes involved in adipogenic program (b) and mitochondrial biogenesis (c) in cells described in a. Data are presented as mean \pm SEM $(n=3)$. d, UCP-1 immunohistochemical staining on a tissue derived from implantation of BMP-7-treated C3H10T1/2 cells into nude mice. Original magnification $=400$ $\mathrm{X}$. 


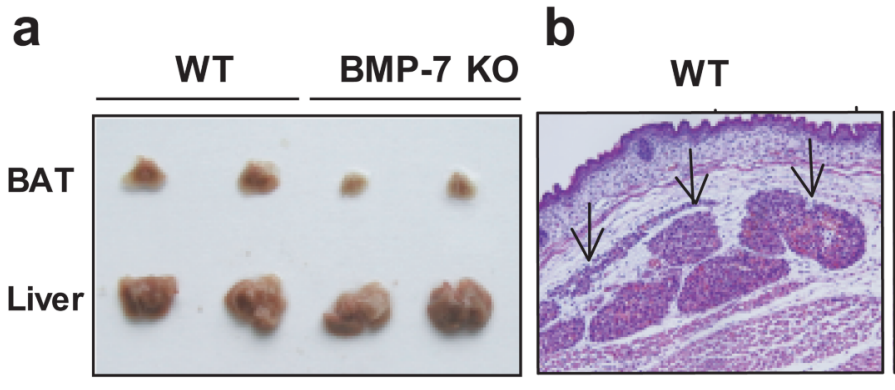

d $\square$ Ad-Lac Z $\square$ Ad-BMP-3 $\square$ Ad-BMP-7

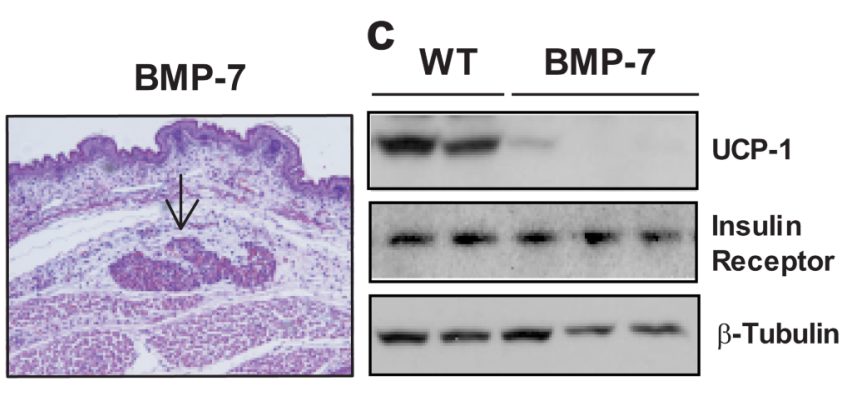

e $\square$ Ad-Lac Z
Ad-BMP-7
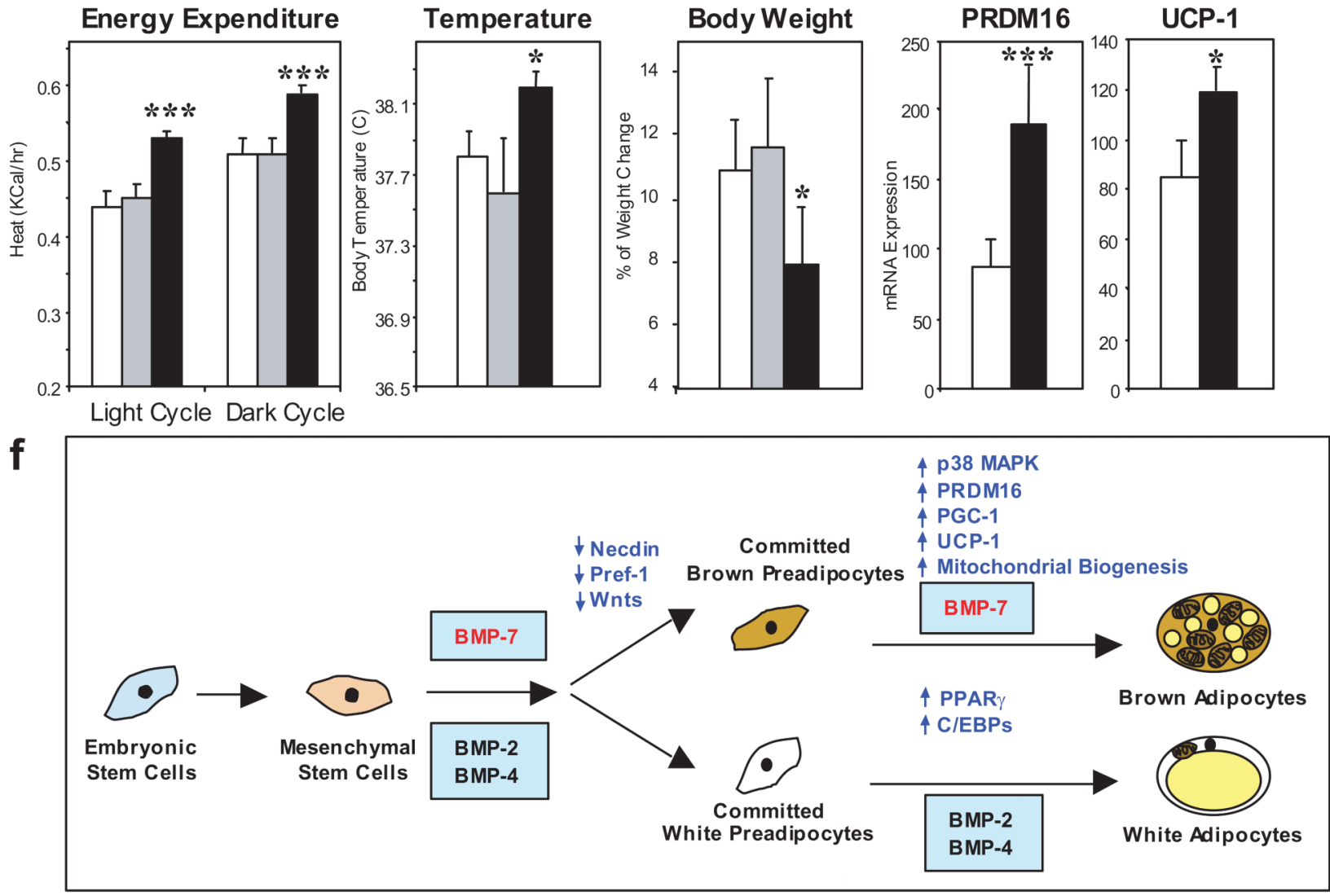

Figure 4. Evidence for an essential role of BMP-7 in BAT development and regulation of whole body energy expenditure by loss-of-function and gain-of-function approaches

a, Gross morphological analysis of BAT and liver from 1-day old wild-type and BMP-7 KO pups. b. Transverse histological sections at the thoracic region from wild-type and BMP-7 KO embryos at $17.5 \mathrm{dpc}$. Slides were stained by $\mathrm{H} \Sigma \mathrm{E}$. Arrows indicate BAT. Original

magnification is $200 \mathrm{X}$. c, Western blotting analysis of UCP-1 and insulin receptor $\beta$ chain in BAT from wild-type and BMP-7 KO embryos at $18.5 \mathrm{dpc}$. $\beta$-tubulin serves as a loading control. d, Adenoviruses expressing BMP-7, BMP-3 or LacZ control were injected into 4-week old C57BL/6 mice via the tail vein $(n=5)$. Energy Expenditure was determined at 10 days after injection by indirect calorimetry. Basal body temperature was measured using a rectal thermometer after 14 days of injection. Percentage of body weight change at day 5 after adenoviral injection was determined by Wilcoxon Signed Rank test. e, Adenoviruses expressing BMP-7 or LacZ control were injected into 12-week old C57BL/6 mice via the tail vein $(n=6)$. Mice were sacrificed 15 days after injection. Expression of PRDM16 and UCP-1 
in BAT was measured by Q-RT-PCR. Data are presented as mean \pm SEM. Asterisks depict statistically significant differences between control and BMP-7 groups $(* P<0.05$, ** $P<$ 0.01 , *** $P<0.001)$. f, Proposed model for the role of BMPs in determination of brown versus white adipocyte development. 